\title{
The Role of B-Type Natriuretic Peptide in the Diagnosis of Congestive Heart Failure in Patients Presenting to an Emergency Department with Dyspnea
}

\author{
Humberto Villacorta, Adriana Duarte, Neison Marques Duarte, Ângela Carrano, Evandro Tinoco Mesquita, \\ Hans J. F. Dohmann, Francisco Eduardo G. Ferreira
}

Rio de Janeiro, RJ - Brazil

\begin{abstract}
Objective - To determine the utility of B-type natriuretic peptide $(B N P)$ in the diagnosis of congestive heart failure (CHF) in patients presenting with dyspnea to an emergency department (ED).
\end{abstract}

Methods - Seventy patients presenting with dyspnea to an ED from April to July 2001 were included in the study. Mean age was $72 \pm 16$ years and 33 (47\%) were male. BNP was measured in all patients at the moment of admission to the ED. Emergency-care physicians, blinded to BNP values, were required to assign a probable initial diagnosis. A cardiologist retrospectively reviewed the data (blinded to BNP measurements) and assigned a definite diagnosis, which was considered the gold standard for assessing the diagnostic performance of BNP.

Results - The mean BNP concentration was higher in patients with CHF $(n=36)$ than in those with other diagnoses $(990 \pm 550$ vs $80 \pm 67 \mathrm{pg} / \mathrm{mL}, \mathrm{p}<0.0001)$. Patients with systolic dysfunction had higher BNP levels than those with preserved systolic function $(1,180 \pm 641 \mathrm{vs} 753 \pm 437 \mathrm{pg} /$ $m L, p=0.03)$. At a blood concentration of $200 \mathrm{pg} / \mathrm{mL}, \mathrm{BNP}$ showed a sensitivity of $100 \%$, specificity of $97.1 \%$, positive predictive value of $97.3 \%$, and negative predictive value of $100 \%$. The application of BNP could have potentially corrected all 16 cases in which the diagnosis was missed by the emergency department physician.

Conclusion - BNP measurement is a useful tool in the diagnosis of CHF in patients presenting to the ED with dyspnea.

Key-words: heart failure, natriuretic peptides, emergency medicine

Hospital Pró-Cardíaco - Unidade de Emergência

Mailing address: Humberto Villacorta - Rua Raimundo Correa, 23/601 - 22040-040

Rio de Janeiro, RJ - Brazil - E-mail: huvillacorta@globo.com
Traditionally, the diagnosis of congestive heart failure (CHF) has been done based on the presence of certain signs and symptoms. In chronic outpatients, in whom the physical signs are usually prominent, the diagnosis of $\mathrm{CHF}$ decompensation is easy. On the other hand, in elderly patients who present to the emergency department (ED) with acute dyspnea, 0 the diagnosis is sometimes challenging. Signs and symptoms may not be sufficiently accurate to make a diagnosis of $\mathrm{CHF}^{1}$. Although the echocardiogram is a good method for diagnosing left ventricular systolic dysfunction, it may not reflect an acute condition ${ }^{2}$. Additionally, as a consequence of population aging, predominant diastolic heart failure is a common finding and thus the presence of a normal systolic function does not rule out the diagnosis of $\mathrm{CHF}$.

B-type (or brain) natriuretic peptide (BNP), which was first isolated from the brain of a monkey, is a neurohormone secreted mainly from the ventricles as a response to volume and pressure overload. BNP promotes diuresis and vasodilation and has been found to be elevated in patients with $\mathrm{CHF}$ and to correlate with New York Heart Association (NYHA) classifications ${ }^{3-7}$.

The aim of this study was to assess the utility of BNP as a biochemical marker of CHF in patients who present to the ED with acute dyspnea.

\section{Methods}

From April to July 2001, 70 consecutive patients who presented to the ED with acute dyspnea were included. Patients with a clear diagnosis of dyspnea, such as trachea stenosis or cardiac tamponade, were excluded as were those with acute coronary syndromes whose prominent complaint was not dyspnea. Besides clinical examination, all patients underwent laboratory tests and chest radiography. Echocardiography was recommended to all patients. Patients with left ventricular fractional shortening equal to or 
less than $25 \%$ were considered as having systolic dysfunction. Whenever necessary, other tests were performed to confirm the diagnosis.

After the initial evaluation, the emergency department physician blinded to BNP measurements was requested to assign an initial diagnosis for the dyspnea. This was compared with the ultimate diagnosis that was performed by a cardiologist who reviewed all patients' data and was also blinded to BNP values. For assessment of the ultimate diagnosis, Boston criteria (definite CHF diagnosis equal to or greater than 8 points), hospital course (response to diuretics and vasodilators, hemodynamic measurements, and others), and test results were taken into account. Patients with both $\mathrm{CHF}$ and other concomitant diagnoses (such as sepsis leading to decompensated $\mathrm{CHF}$ ) were considered as having $\mathrm{CHF}$ as the ultimate diagnosis, because the aim of the study was to assess the ability of BNP in diagnosing CHF. The study was approved by the ethics committee at our hospital and a written postinformed consent was obtained.

BNP measurements - A rapid (15 min) bedside test, which is a fluorescence immunoassay named the Triage BType Natriuretic Peptide test (Biosite Diagnostics Inc., San Diego, California), was used for BNP measurement. A small sample $(5 \mathrm{cc}$ ) of blood was collected into tubes containing potassium EDTA ( $1 \mathrm{mg} / \mathrm{mL}$ of blood). The collection was done by trained technicians and the analysis was processed in whole blood immediately after the collection in the majority of patients. For logistical reasons, a few samples were not analyzed immediately. In these cases, samples were spun down, and the plasma was frozen until the sample was analyzed (1 to 3 days later), an approach known to produce reliable results.

Group comparisons of BNP values were made using the Student $t$ test and analysis of variance (ANOVA). We determined the sensitivity and specificity as well as positive and negative predictive values of BNP for the diagnosis of CHF. We also determined positive and negative likelihood ratios that determine the power of a given test in determining the posttest probability using a pretest probability. The receiver operating characteristic curve was used to establish the best cutoff value of BNP for the diagnosis of CHF. The accuracy was obtained by using the area under the curve. A p value $<0.05$ was considered statistically significant.

\section{Results}

Patients' baseline characteristics are shown in table I. This was the first episode of dyspnea in $15(21 \%)$ patients. Of the 55 (79\%) who had had such a symptom before, 45 (81\%) had a previous diagnosis of dyspnea. Fifty (71\%) patients had dyspnea at rest. Typical signs and symptoms of CHF observed in this population are depicted in table II. As for the medications, $21(30 \%)$ patients were on furosemide, $16(23 \%)$ were on digoxin, $24(34 \%)$ were on angiotensinconverting enzyme inhibitors, and $17(24 \%)$ were on betablockers. Among the latter, only 5 (7\%) were on carvedilol.
The ultimate diagnosis was CHF in $36(51.4 \%)$ cases. Among these patients, coronary artery disease was the cause of CHF in $21(58 \%)$. Five (14\%) were in NYHA class II, 5 were in class III, and $26(72 \%)$ were in class IV. BNP levels for class II, III, and IV were, respectively, $182 \pm 106,544 \pm 324$, e and $994 \pm 675 \mathrm{pg} / \mathrm{mL}$ ( $\mathrm{p}=0.02)$. High quality echocardiograms were obtained in $54(77 \%)$ patients. Among these subjects, $6(11 \%)$ were classified as having systolic dysfunction without acute exacerbation of CHF. Thirty-four $(48.6 \%)$ patients had a diagnosis other than $\mathrm{CHF}$ as the cause of dyspnea.

As shown in figure 1, BNP values were significantly higher in patients with an ultimate diagnosis of CHF than in those with other diagnoses $(990 \pm 550 \mathrm{pg} / \mathrm{mL}$ vs $80 \pm 67 \mathrm{pg} /$ $\mathrm{mL}, \mathrm{p}<0.0001)$. Patients with systolic dysfunction without acute exacerbation of CHF had a mean BNP concentration of $91 \pm 58 \mathrm{pg} / \mathrm{mL}$ (range 22.5 to $196 \mathrm{pg} / \mathrm{mL}$ ). BNP values according to the ultimate diagnoses are shown in table III.

Patients with decompensated CHF due to systolic dysfunction $(n=20)$ had a higher concentration of BNP than those with preserved systolic function $(1,180 \pm 641$ vs $753 \pm 437$

\begin{tabular}{|lc|}
\hline \multicolumn{2}{|c|}{ Table I - Baseline characteristics } \\
\hline Characteristics & Results \\
\hline Age (years) & $72.4 \pm 15.9$ \\
Male gender & $33(47 \%)$ \\
Previous CHF & $26(37 \%)$ \\
COPD & $31(44 \%)$ \\
Renal insufficiency & $6(8,6 \%)$ \\
Systemic hypertension & $36(51 \%)$ \\
Coronary artery disease & $30(42.8 \%)$ \\
Previous acute myocardial infarction & $18(25.7 \%)$ \\
Previous CABG & $14(20 \%)$ \\
Previous PTCA & $7(10 \%)$ \\
Chronic atrial fibrillation & $8(11 \%)$ \\
Left bundle branch block & $14(20 \%)$ \\
Mean blood pressure (mmHg) & $106 \pm 23$ \\
Heart rate (bpm) & $89.2 \pm 18.3$ \\
Serum sodium (mEq/L) & $139.9 \pm 5$ \\
Serum potassium (mEq/L) & $4.2 \pm 0.57$ \\
BUN (mg/dL) & $54 \pm 25.2$ \\
Serum creatinine (mg/dL) & $1.18 \pm 0.6$ \\
C-reactive protein (mmol/L) & $3.6 \pm 5.8$ \\
B-type natriuretic peptide (pg/mL) & $548 \pm 625$ \\
\hline COPD- chronic obstructive pulmonary disease; CABG- coronary artery \\
bypass graft; PTCA- percutaneous transluminal coronary angioplasty; BUN- \\
blood urea nitrogen. \\
\end{tabular}

\begin{tabular}{|ll|}
\hline Table II - Signs and symptoms of CHF in the study population (N=70) \\
\hline Signs and symptoms & N (\%) \\
\hline Rest dyspnea & $50(71 \%)$ \\
Orthopnea & $18(25 \%)$ \\
Paroxysmal nocturnal dyspnea & $16(22.8 \%)$ \\
Edema & $18(25.7 \%)$ \\
Rales & $57(81.4 \%)$ \\
Wheeze & $13(18.5 \%)$ \\
Jugular venous pressure elevation & $19(27 \%)$ \\
S3 & $11(15.7 \%)$ \\
\hline
\end{tabular}


$\mathrm{pg} / \mathrm{mL}, \mathrm{p}=0.03)$. Of note, BNP was significantly higher in the latter group than in those patients without a final diagnosis of $\mathrm{CHF}(753 \pm 436$ vs $80 \pm 67, \mathrm{p}<0.0001)$. Patients admitted from the ED to the hospital $(n=56)$ had a higher concentration of BNP than those notadmitted $(674 \pm 638$ vs $41.6 \pm 54, \mathrm{p}<0.001)$.

A cutoff value of $200 \mathrm{pg} / \mathrm{mL}$ for BNP was the most accurate for the diagnosis of $\mathrm{CHF}$ as the cause of dyspnea. Based on this value, BNP showed a sensitivity of $100 \%$, specificity of $97 \%$, positive predictive value of $97.3 \%$, and negative predictive value of $100 \%$. Positive and negative likelihood ratios were respectively 33 and 0 . The accuracy (area under the curve) was 0.99 .

Nine (13\%) patients initially diagnosed as having decompensated heart failure actually had another diagnosis as the cause of dyspnea. Mean BNP concentration in these patients was $62.6 \pm 42 \mathrm{pg} / \mathrm{mL}$. Conversely, 7 (10\%) patients with an ultimate diagnosis of $\mathrm{CHF}$ were not diagnosed correctly by the emergency department physician. BNP levels in this group were $1,102 \pm 684 \mathrm{pg} / \mathrm{mL}$. If a cutoff value of $200 \mathrm{pg} / \mathrm{mL}$ had been applied to these patients, all $16 \mathrm{mis}-$ diagnosed cases would have been corrected.

\section{Discussion}

The natriuretic peptide system comprises 3 peptides, the A-type peptide (ANP, released from the atria), the B-type peptide (BNP, released primarily from the ventricles), and the C-type peptide (CNP, released from endothelial cells). Because BNP is released directly from the ventricles, it has been suggested that it is more accurate than other natriuretic

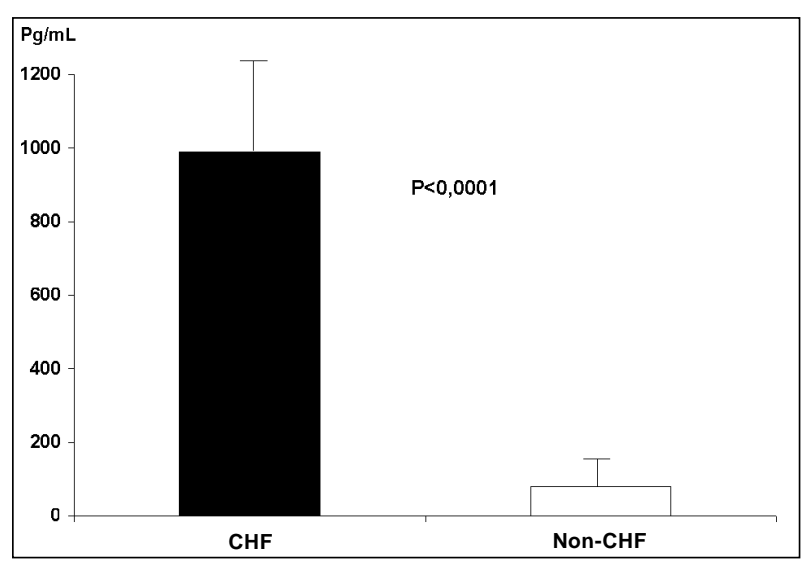

Fig. 1 - BNP levels in patients with and without CHF as a final diagnosis. CHF congestive heart failure.

\begin{tabular}{|lrc|}
\hline \multicolumn{2}{|c|}{ Table III - B-type natriuretic peptide levels according to the ultimate } \\
diagnoses
\end{tabular}

B-type natriuretic peptide in the diagnosis of heart failure

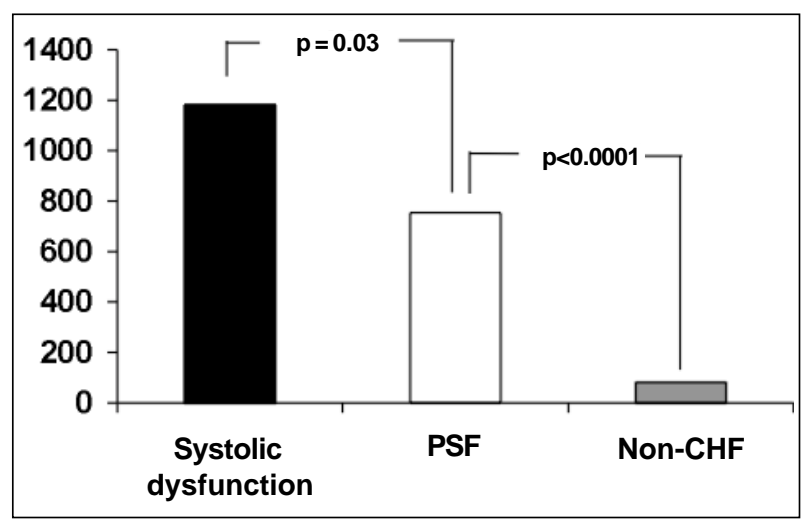

Fig. 2 - BNP levels in patients with CHF due to systolic dysfunction $(\mathrm{n}=20)$, in patients with preserved systolic function (PSF $[n=16]$ ), and patients without CHF as a final diagnosis (non-CHF $[\mathrm{n}=34]$ ).

peptides for the diagnosis of $\mathrm{CHF}^{6,7}$. Like ANP, it promotes diuresis, natriuresis, peripheral vasodilation, renin angiotensin-aldosterone system inhibition, and sympathic nervous system inhibition. BNP is a 32-aa polypeptide, containing a 17-aa structure common to all natriuretic peptides. The nucleic acid sequence of the BNP gene contains the destabilizing sequence "tatttat," which suggests that turnover of BNP messenger RNA is high and that BNP is synthesized in bursts (differently from ANP, which is synthesized and then stored in granules within the atria) ${ }^{8}$. These findings taken together with the fact that BNP has a very short half-life (only $22 \mathrm{~min})^{6}$, suggest that BNP is a cardiac emergency neurohormone, and thus reflects the current moment of ventricular overload. Of interest, BNP correlates well with New York Heart Association classifications and pulmonary wedge pressure modifications during the hospital treatment of patients with decompensated CHF, decreasing in average $33 \mathrm{pg} / \mathrm{mL} / \mathrm{h}$ within the first 24 hours of treatment ${ }^{9}$. BNP failing to decrease with treatment indicates a poor prognosis ${ }^{10}$. Recently, its prognostic value was also demonstrated in patients with acute coronary syndromes ${ }^{11}$.

Comparison with other studies - We demonstrated that BNP measurements can be useful in the diagnosis of $\mathrm{CHF}$ in patients presenting to the ED with dyspnea. This is the first study on this field in our country. Only 1 previous work ${ }^{12}$ assessed the utility of BNP in this scenario. In this study, Dao et al ${ }^{12}$ evaluated 250 patients with acute dyspnea, using a protocol similar to ours. BNP levels in patients with a final diagnosis of CHF were significantly higher than in the non-CHF group $(1,076 \pm 138$ vs $38 \pm 4 \mathrm{pg} / \mathrm{mL})$. Based on a cutoff value of $80 \mathrm{pg} / \mathrm{mL}, \mathrm{BNP}$ was an accurate predictor of a $\mathrm{CHF}$ diagnosis (positive predictive value of $95 \%$ ) and showed excellent accuracy to rule out a diagnosis of CHF (negative predictive value of $98 \%$ ). The general accuracy as assessed by the area under the curve was 0.97 . Of interest, BNP performance was better than that of emergency department physicians (area under the curve of 0.88). The application of BNP with the cutoff suggested above would have potentially corrected 29 of 30 misdiagnosed cases.

Some other authors have assessed the utility of BNP in the primary care setting ${ }^{13-15}$. Davis et al ${ }^{14}$ studied BNP 
and ANP in 52 patients with dyspnea and observed that $\mathrm{BNP}$ was more accurate than ejection fraction or ANP for predicting the diagnosis of CHF.

Our findings are in accordance with those from the study by Dao et al ${ }^{12}$ and confirm the utility of BNP in this scenario. Taken together, these data are overwhelming, because it is very rare to have a method with excellent sensitivity and specificity at the same time. In our study, however, the cutoff value with the most accuracy was $200 \mathrm{pg} / \mathrm{mL}$, which is higher than the cutoff value found by Dao et al ${ }^{12}$. We believe that this difference could be accounted for by the characteristics of our population. We know that the accuracy of a given method depends on disease prevalence and severity. The prevalence of CHF in our study was higher than that in Dao et al's study ${ }^{12}(51.4 \%$ vs $38.8 \%, \mathrm{p}=0.07)$. Likewise, some signs indicated that our patients had more severe disease. For instance, our patients were older than those in the study by Dao et al $(72.4 \pm 15.9$ vs $63 \pm 0.9$ years, $\mathrm{p}<0.001)$. Because BNP correlates with age ${ }^{6}$, the values were probably higher in our population as a whole, and thus the cutoff value was shifted upwards. Additionally, our patients showed a higher rate of hospital admission $(56.8 \%$ vs $20 \%, \mathrm{p}<0.0001)$. We also would like to point out that $86 \%$ of our patients with CHF were in NYHA class III or IV and, therefore, a higher cutoff value would be more suitable for identifying these patients. We must emphasize, however, that a cutoff value of $80 \mathrm{pg} / \mathrm{mL}$ is more accurate in a nonselected or outpatient population, in which the number of individuals in class I or II is usually higher.

Two large population-based studies sought to investigate the utility of BNP in the diagnosis of left ventricular systolic dysfunction. In 1 study carried out in Glasgow, Scotland ${ }^{16}, 1,653$ subjects were included and the accuracy of BNP to predict left ventricular systolic dysfunction on the echocardiogram was assessed. BNP less than $18 \mathrm{pg} / \mathrm{mL}$ showed an excellent negative predictive value of $97 \%$. The area under the curve was $95 \%$.

In an unpublished study (Heart Failure Society of America, Washington DC, USA, September 2001), Redfield et al investigated 2,042 subjects in Omsted. BNP was very accurate in detecting systolic dysfunction (area under the curve showed a $95 \%$ confidence interval of 0.85 to 0.97 ). In this study, the accuracy of BNP for detecting diastolic dysfunction was also investigated. The prevalence of diastolic dysfunction in the entire population was $6.7 \%$, and $80 \%$ of these subjects were asymptomatic. A restrictive or pseudo-normal pattern on the echocardiogram was used as the gold standard for the diagnosis of diastolic dysfunction. The area under the curve for BNP to detect diastolic dysfunction was 0.79 .

Study limitations - This study was carried out in a single center that is a referral center for cardiology in our city. Therefore, one must be cautious when generalizing our data. It is possible that in general hospitals, the best cutoff value to detect decompensated CHF may be different. A multicenter trial named "Breathing Not Properly in Congestive Heart Failure" is underway to address this issue in a broader population.

We conclude that BNP measurement is a sensible and specific strategy for diagnosing $\mathrm{CHF}$ in patients who present to the ED with acute dyspnea and may add additional benefits to the management of patients with CHF in this setting.

\section{References}

1. Stevenson LW. The limited availability of physical signs for estimating hemodynamics in chronic heart failure. JAMA 1989; 261: 884-8.

2. Deveraux RB, Liebson PR, Horan MJ. Recommendations concerning use of echocardiography in hypertension and general population research. Hypertension 1987; 9: 97-104.

3. Dicstein K. Natriuretic peptides in detection of heart failure. Lancet 1998; 35:3-4.

4. Maeda K, Takayoshi T, Wada A, Hisanaga T, Kinoshita M. Plasma brain natriuretic peptide as a biochemical marker of high left ventricular end-diastolic pressure in patients with symptomatic left ventricular dysfunction. Am Heart J 1998; 135: 825-32.

5. Clerico A, Iervasi G, Chicca M, et al. Circulating levels of cardiac natriuretic peptides (ANP and BNP) measured by highly sensitive and specific immunoradiometric assays in normal subjects and in patients with different degrees of heart failure. J Endocrinol Invest 1998; 21: 170-9.

6. Maisel A. B-type natriuretic levels: a potential novel "White count" for congestive heart failure. J Cardiac Fail 2001; 7: 183-93.

7. Brandt RR, Burnett Jr JC. Humoral control of the kidney during congestive heart failure: role of cardiac natriuretic peptides and angiotensin II. In: Poole-Wilson PA, Colucci WS, Massie BM, Chattenjee K, Coats AJS, eds. Heart Failure. New York: Churchill Livingstone, 1997: 143-54.

8. Nagagawa O, Ogawa Y, Itoh H, Suga S, Komatsu Y, Kishimoto I. Rapid transcriptional activation and early mRNA turnover of BNP in cardiocyte hypertrophy: evidence for BNP as an "emergency" cardiac hormone against ventricular overload. J Clin Invest 1995; 96: 1280-7.

9. Kazanagra R, Cheng V, Garcia A. A rapid test for B-type natriuretic peptide correlates with falling wedge pressures in patients treated for decompensated heart failure: a pilot study. J Cardiac Fail 2001; 7: 21-9.

10. Cheng V, Kazanagra R, Garcia A, et al. A rapid bedside test for B-type peptide predicts treatment outcomes in patients admitted for decompensated heart failure: a pilot study. J Am Coll Cardiol 2001; 37: 386-91.

11. de Lemos JA, Morrow DA, Bentley JH, et al. The prognostic value of B-type natriuretic peptide in patients with acute coronary syndromes. N Engl J Med 2001; 345: 1014-21.

12. Dao Q, Krishnaswamy P, Kazanegra R, et al. Utility of B-type natriuretic peptide in the diagnosis of congestive heart failure in an urgent-care setting. J Am Coll Cardiol 2001; 37: 379-85.

13. Cowie MR, Struthers AD, Wood DA, et al. Value of natriuretic peptides in assessment of patients wtih possible new heart failure in primary care. Lancet 1999; 350: 1347-51.

14. Davis M, Espiner E, Richards G, et al. Plasma brain natriuretic peptide in assessment of acute dyspnea. Lancet 1994; 343: 440-4.

15. Koon J, Hope J, Garcia A, et al. A rapid bedside test for brain natriuretic peptide accurately predicts cardiac function in patients referred for echocardiography. $\mathrm{J}$ Am Coll Cardiol 2000; 35: 419A.

16. McDonagh TA, Robb SD, Murdoch DR, et al. Biochemical detection of leftventricular systolic dysfunction. Lancet 1998; 351: 13. 